\title{
Multiple-Scattering Noise in One Dimension: Universality through Localization-Length Scaling
}

\author{
Ping Sheng, Zhao-Qing Zhang, ${ }^{(a)}$ Benjamin White, and George Papanicolaou ${ }^{(b)}$ \\ Corporate Research Science Laboratories, Exxon Research \& Engineering Company, Annandale, New Jersey 08801
}

(Received 16 May 1986)

\begin{abstract}
We present a study of nonstationary multiple-scattering noise resulting from pulse reflection from a one-dimensional randomly layered half-space. It is shown that the noise power spectrum exhibits model-independent universal behavior when it is analyzed in terms of a single dimensionless variable $x$, defined as the ratio between the mean distance traveled by the pulse and the frequency-dependent localization length. Results of numerical simulation indicate that the spectrum is a peaked function of $x$, where the height of the peak defines the expected value of a noise upper bound.

PACS numbers: $43.50 .+\mathrm{y}, 03.40 . \mathrm{Kf}, 63.50 .+\mathrm{x}$
\end{abstract}

The use of a transient pulse as a material or target probe is a technique that has both industrial and geophysical applications. When the probed or the intervening medium contains randomness, the timedependent reflection and transmission signals are generally characterized by the presence of noise arising from multiple scattering of the pulse. A good example of this can be found in the complex signal tail, known as coda, ${ }^{1}$ following the arrival of each seismic pulse after it has traversed the inhomogeneous earth. While the structure of this tail follows deterministically from the structure and properties of the earth, the possibility of recovering this type of information from the coda is nevertheless rather remote since the information is thoroughly scrambled by multiple scattering. The question is then what information, if any, can be extracted from this type of noise? In this work we study the reflection noise resulting from an acoustic or electromagnetic pulse propagation and scattering through a one-dimensional randomly layered medium. It is shown that the nonstationarity of the noise has a continuous range of time scales defined by the frequency-dependent localization length ${ }^{2-5} l(\omega)$ divided by the mean velocity $v_{0}$. When the observation time $\tau$ is scaled in terms of $l(\omega) / v_{0}$, the noise-power spectrum is demonstrated to be a universal function of the scaled variable $x \equiv \tau v_{0} / l(\omega)$ independent of model parameters. For low frequencies, this universal character of the spectral function is in fact supported by the earlier mathematical work of Burridge, Papanicolaou, and White. ${ }^{6}$ The identification of localization length $l(\omega)$ as the scaling quantity generalizes the theory and extends the domain of universality to all frequencies. Through numerical simulations we have also determined the characteristics of the universal spectrum function in both the time and the frequency domains. In our present case the answer to the questions posed earlier is therefore that, with the knowledge of the universal spectrum function, the maximum information that one can directly extract from the noise would be the localization length as a function of frequency. In addition, the knowledge about the character of the noise also opens the possibility for enhancing signals, i.e., scatterings from target objects, through noise suppression.

Consider a one-dimensional randomly layered halfspace in the region $0 \leqslant z<\infty$ characterized by spatially varying density $\rho(z)$, elastic modulus $K(z)$, dielectric constant $\epsilon(z)$, and magnetic permeability $\mu(z)$. Since the acoustic and the electromagnetic problems are related by a mapping of the variables, ${ }^{7}$ the results obtained in one case are assured to be applicable in the other case as well. In the following we will consider the acoustic problem. The density and elastic-modulus variations are modeled by piecewise-constant profiles

$$
\begin{aligned}
& \rho_{i}=\rho_{0}\left[1+\sigma_{\rho} N_{i}\right], \\
& K_{j}^{-1}=K_{0}^{-1}\left[1+\sigma_{K} N_{j}\right],
\end{aligned}
$$

where $i$ (or $j$ ) denotes $i$ th (or $j$ th) piecewise layer, $0 \leqslant \sigma_{\rho(K)}<1$ specifies the amount of randomness, and $N_{i}$ is a random number with a uniform distribution in the interval $[-1,1]$. The thickness of the layers can be either constant, denoted as model I, or variable with an exponential distribution, denoted as model II. In the region $-\infty<z<0$ we will assume the medium to be homogeneous with $\rho=\rho_{0}, K=K_{0}$, and mean velocity $v_{0}=\left(K_{0} / \rho_{0}\right)^{1 / 2}$. The initial condition of the problem is that beginning at $\tau=0,{ }^{8}$ a pulse moving from left to right with velocity $v_{0}$ is incident on the random medium. For an observer sitting at $z=0$, the response of the medium at time $\tau$ is given by

$$
Q(\tau)=(1 / 2 \pi) \int_{-\infty}^{\infty} d \omega \exp (i \omega \tau) f(\omega) R(\omega),
$$

where $Q$ can be either the pressure or the displacement velocity, $f(\omega)$ denotes the amplitude of the pulse frequency component, and $R(\omega)$ is the reflection coefficient for a single-frequency wave. The time correla- 
tion function of the reflected signal can now be written as

$$
\begin{aligned}
\langle Q(\tau) Q(\tau+t)\rangle & =\frac{1}{(2 \pi)^{2}} \int_{-\infty}^{\infty} d \omega_{1} \int_{-\infty}^{\infty} d \omega_{2} f\left(\omega_{1}\right) f^{*}\left(\omega_{2}\right) \exp \left(-i \omega_{2} t\right)\left\langle R\left(\omega_{1}\right) R^{*}\left(\omega_{2}\right)\right\rangle \exp \left[i\left(\omega_{1}-\omega_{2}\right) \tau\right] \\
& =\frac{1}{2 \pi} \int_{-\infty}^{\infty} d \omega \exp [-i \omega t]\left[\frac{1}{2 \pi} \int_{-\infty}^{\infty} d h f\left(\omega-\frac{h}{2}\right) f^{*}\left(\omega+\frac{h}{2}\right) \exp \left(-i h \tau^{\prime}\right) U(h, \omega)\right]
\end{aligned}
$$

where the angular brackets denote configurational averaging, $\omega=\left(\omega_{1}+\omega_{2}\right) / 2$ is the center frequency, $h=\omega_{2}-\omega_{1}, \tau^{\prime}=\tau+(t / 2)$, and

$$
U(h, \omega)=\left\langle R(\omega-h / 2) R^{*}(\omega+h / 2)\right\rangle .
$$

It is easily seen from Eq. (3) that the quantity in the square brackets is exactly the power spectrum of the reflected signal $S(\tau, \omega)$. If $U(h, \omega)=c \delta(h)$, then this spectrum is given by $c|f(\omega)|^{2}$, i.e., the noise is white and stationary. However, in reality we expect $U(h, \omega)$ to have a small but finite width $\Delta h<<\omega$ with $U(h=0, \omega)=1 \quad$ (localization of waves implies $|R|=1)$, and $U(-h, \omega)=U^{*}(h, \omega)$. Since $h$ is the conjugate variable to $\tau, \Delta h$ in essence determines the time scale of nonstationary for the reflected noise. Given that $U(h, \omega)$ is nonzero only for $|h| / \omega<<1$, we can write the spectrum as

$$
S(\tau, \omega) \cong|f(\omega)|^{2} \bar{U}(\tau, \omega),
$$

where

$$
\bar{U}(\tau, \omega)=(1 / 2 \pi) \int_{-\infty}^{\infty} d h \exp (-i h \tau) U(h, \omega)
$$

is the noise spectrum function.

To calculate the function $U(h, \omega)$, we use the transfer-matrix method ${ }^{2}$ to evaluate $R(\omega \pm h / 2)$ at different center frequencies $\omega$ and different values of $h$. For the one-dimensional acoustic problem, $R(\nu)=-q^{*}(\nu) / p^{*}(\nu)$, where $p$ and $q$ are given by

$$
\left(\begin{array}{cc}
p & q \\
q^{*} & p^{*}
\end{array}\right)=\prod_{i=1}^{N} T_{i, i-1},
$$

with $T_{i+1, i}$ denoting a $2 \times 2$ matrix with elements

$$
\begin{aligned}
& t_{11}=t_{22}^{*}=0.5\left[1+\left(\rho_{i+1} K_{i+1} / \rho_{i} K_{i}\right)^{1 / 2}\right] \exp \left[i \nu\left(\phi_{i+1}+\phi_{i}\right)\right], \\
& t_{12}=t_{21}^{*}=0.5\left[1-\left(\rho_{i+1} K_{i+1} / \rho_{i} K_{i}\right)^{1 / 2}\right] \exp \left[i \nu\left(\phi_{i+1}-\phi_{i}\right)\right],
\end{aligned}
$$

and $\phi_{i}=a_{i}\left(\rho_{i} / K_{i}\right)^{1 / 2} / 2$. The value of $N$ is chosen to be large enough so that $|R|=1$ and the phase angle of $R$ stabilizes. In Fig. 1 we show the real and imaginary parts of $U$, averaged over 400 configurations, for two models, in which model $I$ has fixed layer thickness $a_{i}=1, \rho_{0}=8, K_{0}=2, \sigma_{\rho}=0.05, \sigma_{K}=0.4$, and model II has the parameter values $\rho_{0}=K_{0}=1, \sigma_{\rho}=\sigma_{K}=0.9$, and the probability distribution of layer thicknesses $P\left(a_{i}\right)=\exp \left(-a_{i}\right)$. The data for a total of fifteen center frequencies for model I and eleven center frequencies for model II are shown. In addition, the solid lines denote the Fourier transforms of the timedomain simulation data to be explained later. For each center frequency $\omega$, the values of $U$ are plotted as a function of $\ln y$, where $y \propto h$ is a dimensionless variable obtained by scaling of $h$ in terms of $v_{0}$ divided by a scaling length $l(\omega)$, i.e., $y=h l(\omega) / v_{0}$. It is seen, first of all, that $U$ indeed possesses a finite width as expected. Moreover, by adjustment of the value of $l(\omega)$ for each center frequency $\omega$ all the data points in terms of $y$ fall within a band whose width is defined by statistical fluctuations. This fact gives strong indication that $U$ is a function of a single variable $y$. Furthermore, $U$ is model independent since completely different random models yield the same functional form (this fact has been verified for more than the two types of models described above). These items of evidence, coupled with a mathematical proof of the singlevariable and model-independent properties of $U$ in the low-frequency limit ${ }^{6}$ [(mean layer thickness) $\left.\times \omega / v_{0} \rightarrow 0\right]$, lead us to believe that $U$ is indeed a universal function. ${ }^{9}$

In Fig. 2 we show the values of $l(\omega)$ used to obtain the scaling variable $y$ in Fig. 1 . On the same graph we also plot the localization length ${ }^{4}$ calculated independently as $\left\langle-\ln \left[1-|R(z)|^{2} / 2 z\right\rangle^{-1}\right.$ in the limit of large $z$, for the same two models. The remarkable tracking of the two curves, especially in the region of undulating variations $\mathrm{s}^{10}$ for model $\mathrm{I}$, leaves no doubt that $l(\omega)$ is in fact the localization length. It should be noted that the range of frequency covered is large, ranging from $\lambda=2 \pi v_{0} / \omega=20 \bar{a}$ to $\lambda=0.5 \bar{a}$, where $\bar{a}$ is the mean layer thickness. This fact tells us that the scaling relation is valid in practically all frequency ranges provided that one uses the localization length as the scaling quantity. Also, since the width of the function $U(y)$ is $\Delta y=\Delta h l(\omega) / v_{0}=1$, the time scale of noise nonstationarity can be deduced to be $(\Delta h)^{-1}$ $=l(\omega) / v_{0}$. Besides verifying the earlier assumption of 


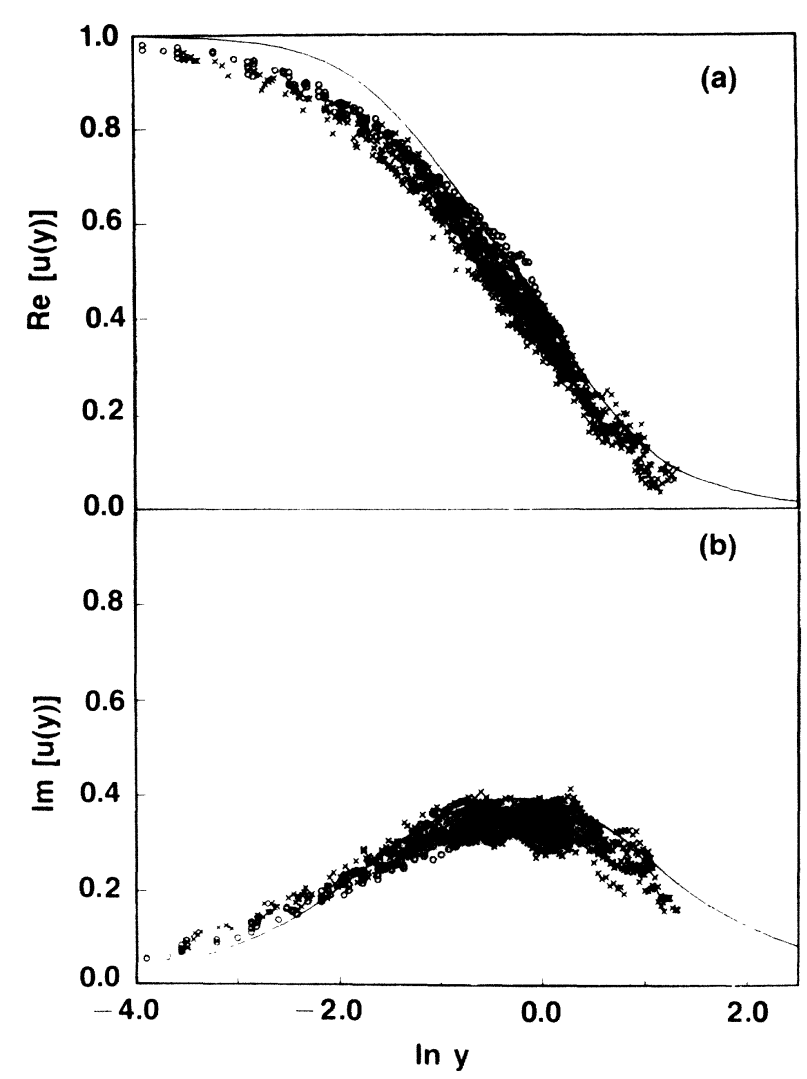

FIG. 1. (a) The real part and (b) imaginary part of the function $U(y)$ as functions of $\ln y$, where $y=h l(\omega) / v_{0}$. Crosses denote the results of model I; open circles those of model II.

$\Delta h / \omega=v_{0} / \omega l(\omega)<<1$, from the behavior of $l(\omega)$ as a decreasing function of frequency (seen in Fig. 2) we predict that the low-frequency components of the noise will persist longer, and the noise frequency content should therefore continuously shift downward at long observation times.

The scaling property of $U$ directly implies that the noise spectrum function $\bar{U}(\tau, \omega)$, Eq. (6), should be expressible as

$$
\bar{U}(\tau, \omega)=\mu(x) / \tau,
$$

where $x=\tau v_{0} / l(\omega)$ and $^{11}$

$$
\mu(x)=\left\{\begin{array}{l}
(x / 2 \pi) \int_{-\infty}^{\infty} U(y) \exp (i x y) d y, \quad x \geqslant 0, \\
0, \quad x<0 .
\end{array}\right.
$$

Since $\bar{U}$ has the dimension of [time] ${ }^{-1}, \mu(x)$ is noted to be dimensionless. Moreover, by using the inverse Fourier transform of $\mu(x)$ to $U(y)$ and the fact that $U(0)=1$, we derive a sum rule for $\mu(x)$ :

$$
\int_{0}^{\infty} x^{-1} \mu(x) d x=1 \text {. }
$$

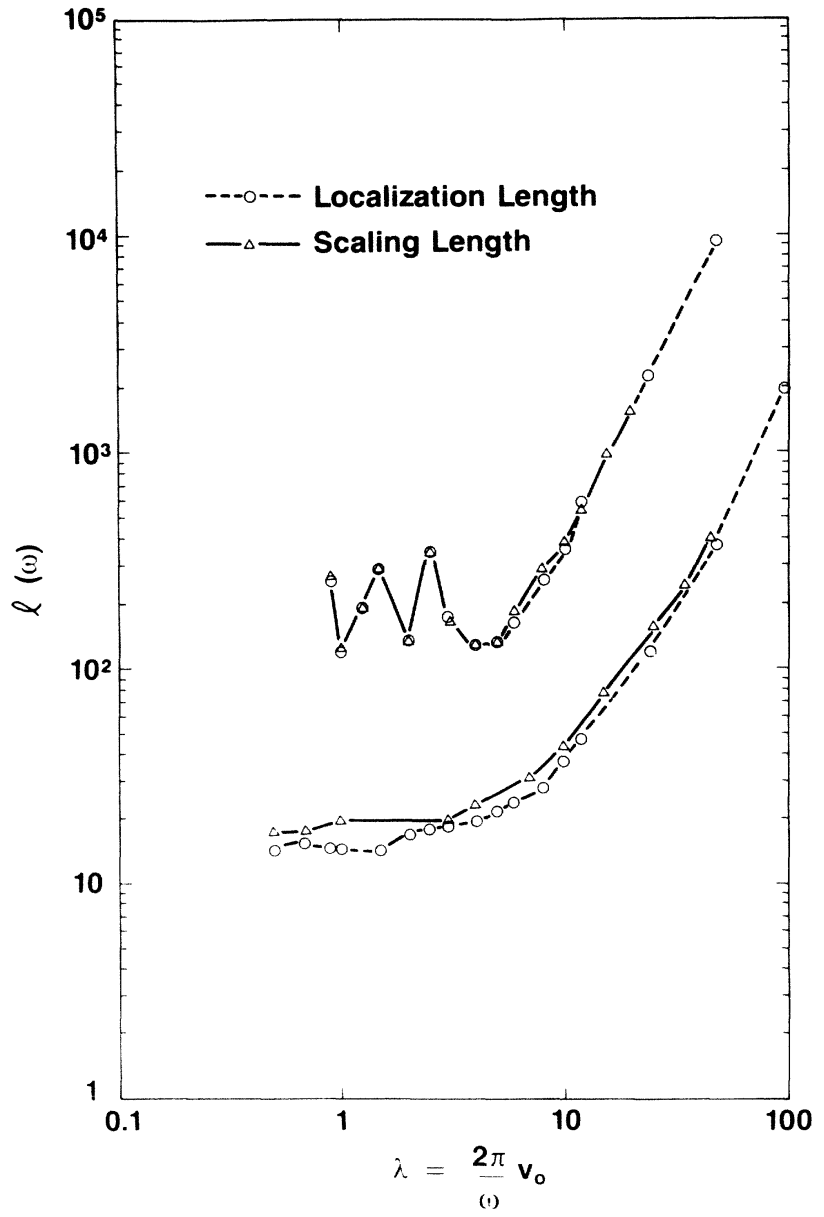

FIG. 2. The scaling length $l(\omega)$ and the localization length as functions of wavelength $\lambda=2 \pi v_{0} / \omega$, both in units of mean layer thickness $\bar{a}$. Results for model I are shown in the upper pair of curves, and those for model II are shown in the lower pair. The agreement between the two quantities indicates that $l(\omega)$ can be identified as the localization length. Note the asymptotic $\omega^{-2}$ dependence at low frequencies.

To obtain the function $\mu(x)$, we have carried out simulations in the time domain by solving the acoustic wave equation,

$$
[K(z)]^{-1} \ddot{Q}=\partial\left\{[\rho(z)]^{-1} \partial Q / \partial z\right\} / \partial z,
$$

numerically for 900 configurations of model I with a Gaussian incident pulse. By Fourier transformation of $\langle Q(\tau) Q(t+t)\rangle$ with respect to $t$, we obtain $S(\tau, \omega)$ for five different observation times $\tau$. The values of $\mu(x)$ shown in Fig. 3 are evaluated as $S(\tau, \omega) \tau /$ $|f(\omega)|^{2}$ and plotted as a function of $\sqrt{x}$ by use of independently calculated values of the localization length. We use $\sqrt{x}$ as the plotting variable because at low frequencies $\sqrt{x} \propto \omega$, which makes the plotted 


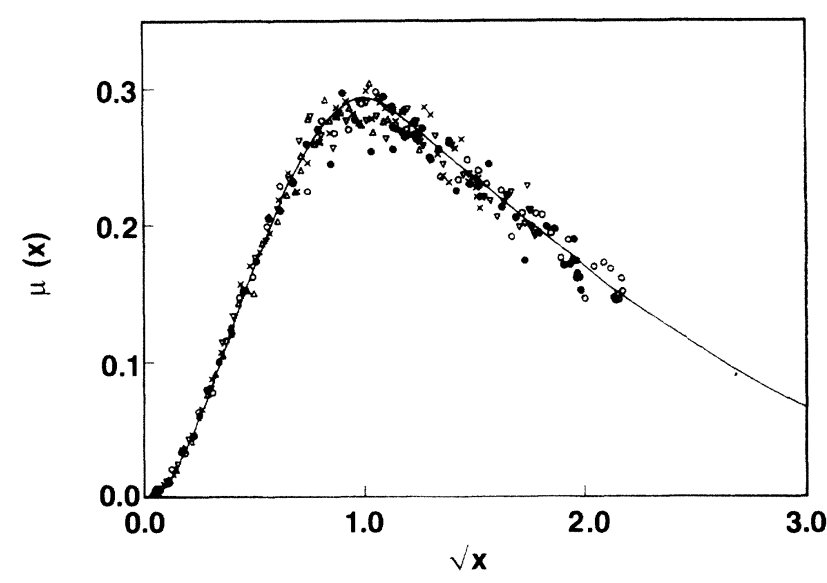

FIG. 3. The noise spectrum function $\mu(x)$ as a function of $\sqrt{x}$, where $x=\tau v_{0} / l(\omega)$. In time units of (mean layer thickness) $/ v_{0}$, noise data for $\tau=200,300,400,500$, and 600 are shown.

curve proportional to the noise-frequency spectrum in that limit.

Several features and implications of Fig. 3 should be noted. First of all, the predicted scaling property of $\mu(x)$ is verified in that the data for different $\tau$ 's collapse into a single curve after the localization-length scaling. Second, $\mu(x)$ has a peak at $x \simeq 1$, where the height of the peak, $\mu^{*}(\simeq 0.3)$ defines $\mu^{*} / \tau$ as the expected value of an upper bound for the noise power. A physical interpretation of the peak is that it separates the effective-medium regime ${ }^{12}$ for $x<1$, where the wave does not yet see the localization character of the medium, from the wave-scattering regime for $x \geqslant 1$, where the characteristic behavior is dominated by exponential decay of the wave amplitudes. Third, since $l(\omega)$ has a lower bound ${ }^{4} L$ for a given randomness model, a minimum pulse traveling time $\tau=L / \nu_{0}$ is required before the condition of maximum noise, $x=1$, can be reached. Last, by drawing a line through the data points and then fitting an exponential tail to the curve, we verify that the sum rule, Eq. (9), can be satisfied by only slightly adjustment of the rate of the tail decay. The Fourier transform of $x^{-1} \mu(x)$ then yields the solid lines shown in Fig. 1. The excellent overall accord with the frequency-domain data clearly offers a verification for the internal consistency of the theory.

In conclusion, we have presented a framework for the analysis of multiple-scattering noise that consists of a universal part, which is a manifestation of the generic multiple-scattering effect, and a modeldependent part, expressed by the localization length. ${ }^{13}$ Application of the theory, plus the effect of higher spatial dimension, are presently under consideration.

(a) On leave from San Francisco State University, 1600 Halloway, San Francisco, CA 94132, and Institute of Physics, Academia Sinica, People's Republic of China.

(b) Permanent address: Courant Institute of Mathematical Sciences, 251 Mercer St., New York, NY 10012.

1P. C. Richards and W. Menke, Bull. Seismol. Soc. Am. 73, 1005 (1983).

2M. Ya. Azbel, Phys. Rev. B 28, 4106 (1983).

${ }^{3}$ S. John, H. Sompolinsky, and M. J. Stephen, Phys. Rev. B 27, 5592 (1983).

4P. Sheng, B. White, Z. Q. Zhang, and G. Papanicolaou, Phys. Rev. B (to be published).

${ }^{5 W}$. Kohler and G. Papanicolaou, J. Math. Phys. 14, 1733 (1973).

${ }^{6} \mathrm{R}$. Burridge, G. Papanicolaou, and B. White, to be published.

${ }^{7} \epsilon / c \rightarrow \rho, c / \mu \rightarrow K$, electric field $\rightarrow$ displacement velocity, magnetic field $\rightarrow$ pressure. Here $c$ denotes the speed of light.

$8 \tau=0$ is defined by the instant at which a delta-function pulse hits $z=0$. The response to any other pulse shape can be expressed as a convolution of the pulse with the response to a delta function.

${ }^{9}$ The fact that after the localization-length scaling the functional form of $U(y)$ for high frequencies agrees with that for low properties, where we have mathematical proof of the claimed properties, shows that the model-independent character of $U(y)$ extends beyond the low-frequency limit.

${ }^{10}$ The undulations in the localization length as a function of wavelength (frequency) are typical for equal-layerthickness models. They may be interpreted as remnants of a periodic system.

${ }^{11} \mu(x)=0$ for $x<0$ is a statement of causality.

${ }^{12}$ At $x<<1$ it can be shown that $\mu(x)=2 x$. Therefore, at early observation times ( $\tau$ small) the noise spectrum has the form $\bar{U}=2 x / \tau=2 v_{0} / l(\omega)$, i.e., the noise would seem to be stationary but nonwhite, with the noise-frequency dependence governed by the frequency dependence of (localization length) ${ }^{-1}$.

${ }^{13}$ The localization length expresses the wave attenuation length due to multiple scattering. When there are other dissipation mechanisms present, their effects can be estimated by comparison of the dissipation attenuation length $l_{d}$ with localization length $l$. If $l_{d} \gg l$, then dissipation can be neglected. If not, the net wave attenuation length $L$ is obtainable as $L^{-1}=l_{d}^{-1}+l^{-1}$. The effect of dissipation on our universal function remains to be studied. 